\title{
SAFETY \& EFFICACY OF SUPRACOSTAL ACCESS FOR PCNL : NIKDU EXPERIENCE
}

\author{
BISWAS NP ${ }^{1}$, RASHID MM ${ }^{2}$, ARAFUZZAMAN $K^{3}$, UDDIN MM ${ }^{4}$, ALAM MN ${ }^{5}$
}

\begin{abstract}
Aims and objective : To evaluate the safety and efficacy of supracostal puncture for PCNL procedure.

Materials and methods : This is a retrospective study conducted in Department of Urology, NIKDU, Dhaka, from January 2013 to December 2016. A total of 408 patients underwent PCNL, of whom 56 (13.72\%) had supracostal access. All procedures were performed under $G / A$. All were performed in a single setting \& the data were analysed for indications, stone clearance rates and the complications associated with supracostal access. The indications for supracostal access were staghorncalculi , uppercalyceal stone, pelvic stone, complex inferior calyceal stones, stones in upper ureter and high lying kidney. All supracostal punctures were made in $11^{\text {th }}$ intercostal space and was made by the urologists under $C$ - arm fluoroscopic guidance in prone position. After tract dilatation ultrasound lithotripsy was used for fragmentation of stones. The operative time, success rate, hospital stay and complications were evaluated.
\end{abstract}

Results : Single tract access was used in 44 (78\%) cases, 12 (22\%) patients required a second tract. Additional punctures were needed for staghorn calculi (4 out of 14). Overall $82 \%$ of the patients were rendered stone free or had a clinically insignificant residual calculi with PCNL alone and this increased to $96 \%$ with ancillary procedures. In patient with staghorn calculi, they were completely cleared in $78 \%$. Overall complication rate was $26 \%$ which included hydrothorax in $5(10 \%)$ patients, 3 of which required insertion of chest tube. 2 (3.5\%) patient developed haemothorax due to injury to intercostals vessels, $2(3.5 \%)$ patient developed perforation of the pelvis, 2(3.5\%) developed perinephric collection, infection/ urosepsis in 4(7\%). The rest of the patients recovered successfully. Post operative stay ranged from 2 to 9 days.

Conclusion : Supra costal puncture is safe and effective option which give high stone clearance rate with acceptable complications. With a good understanding of the anatomy surrounding the upper pole of the kidney and attention to a few technical considerations during the procedures, access is very safe and effective.

Bangladesh J. Urol. 2018; 21(2): 140-144

\section{Introduction:}

Percutaneous nephrolithotomy is a feasible option for the treatment of large renal stone burden, staghorn calculi and upper ureteral calculi, lower pole calyceal stones greater than $10 \mathrm{~mm}$ and diverticular stones. ${ }^{1}$

1. Associate Professor, Department of Urology, National Institute of Kidney Diseases \& Urology (NIKDU), Dhaka.

2. Assistant Professor, Mugda Medial College, Dhaka

3. Medical Officer, Department of Urology, National Institute of

4. MS Urology Final Part Student, Department of Urology, National Institute of Kidney Diseases \& Urology (NIKDU), Dhaka.

5. Assistant Professor, Department of Urology, National Institute of Kidney Diseases \& Urology (NIKDU), Dhaka.

Correspondences: Biswas NP, Department of Urology, National Institute of Kidney Diseases \& Urology, Dhaka.

Received: 9 August 2017

Accepted: 05 March 2018
Gaining percutaneous access and entering the appropriate calyx are critical to the procedure. The access directly relates to the ease of the procedure and subsequent success of stone clearance. The anatomy of the kidney often favors an approach through the superior calyx. ${ }^{2}$ Common approach to PCNL access is below the $12^{\text {th }}$ rib, however some cases have better success via a supracostal approach. A subcostal approach can result in suboptimal access and reduced stone clearance.

Usually the upper pole of the kidney is more posterior and more medial than the lower pole, so access via a superior posterior calyx is more direct to the long axis of the kidney. This approach traditionally facilitates the access to a lower calyx and the upper ureter. Supracostal puncture is also useful in obese patients 
and those with high lying kidneys. ${ }^{3}$ Careful preoperative and intraoperative planning is required to determine the renal access for complete stone clearance. This may require a supracostal approach for stone removal.

There are some potential complications of supracostal puncturelike pneumothorax, hydrothorax, and lung injury. ${ }^{4}$ The upper pole is more commonly accessed via a supracostal route, but can be accessed through a subcostal approach. The supracostal approach is less often used because of concerns about safety and thoracic complications. ${ }^{5}$ We evaluated the safety and efficacy of supracostal access for PCNL in selective cases.

\section{Materials and methods :}

Total number of patients who underwent PCNL in the study period was 408 . Of which supracostal approach was necessary in 56 cases.. The decision to adopt supracostalaccess was based on the stone location, configuration of the intrarenal collecting system, and the likelihood of maximal stone clearance.

All patients radiographic images wererevewed. The preoperative assessment included routine laboratory studies, eg, blood biochemistry, CBC, coagulation profile and negative urine culture.

Radiological evaluation was done by X-Ray KUB, USG of KUB, IVU, CT scan. Patients with preoperative positive urine culture received antibiotic for 1 week preoperatively.

All procedures were performed in a single stage.After induction of GA, patient was placed in lithotomy position, ureteral catheter was negotiated. The patient was then turned to prone position, RGP was done to select the appropriate calyx. The puncture was done above the $12^{\text {th }}$ rib in 56 patients, and it was done throughthe lateral half of the intercostal space breathholding in full expiration and the needle was advanced through the middle of the ICS to avoid the vessels.

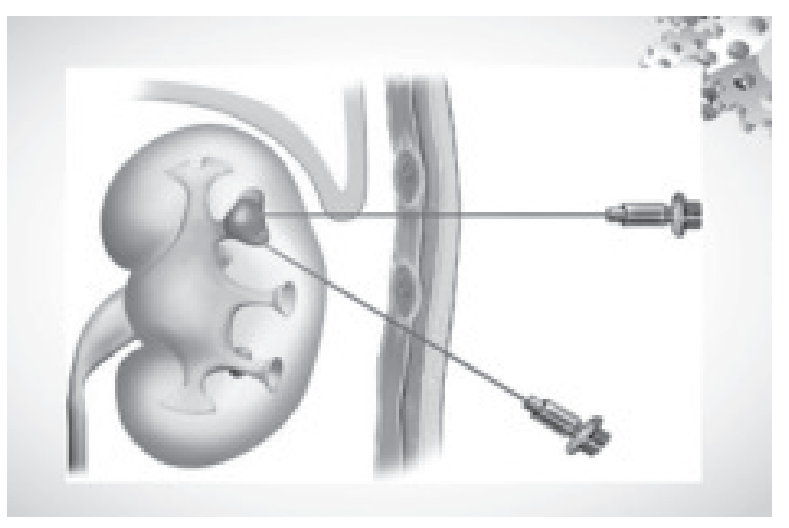

Fig.-1: Puncture site.

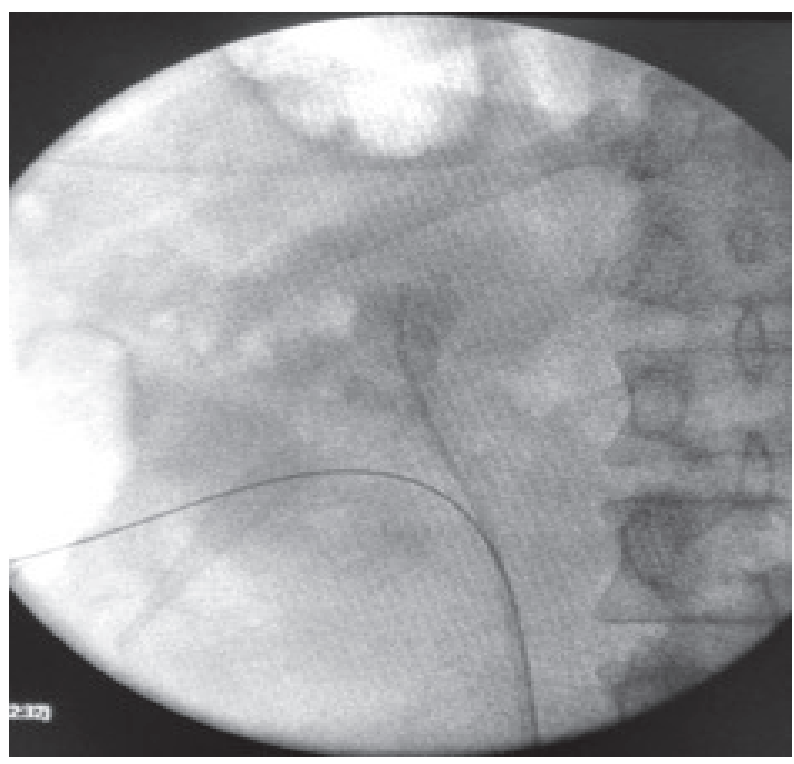

Fig.-3: First guidewire introduced
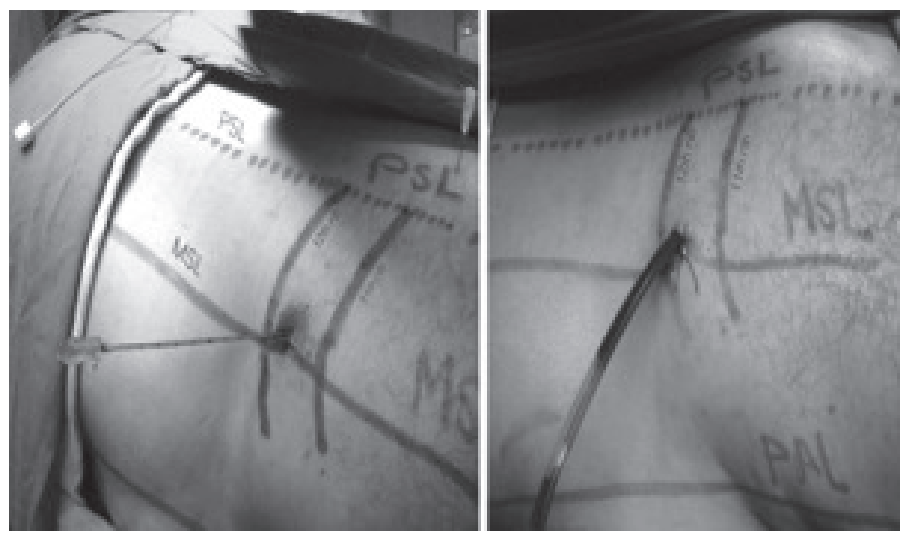

Fig.-2: Skin marking

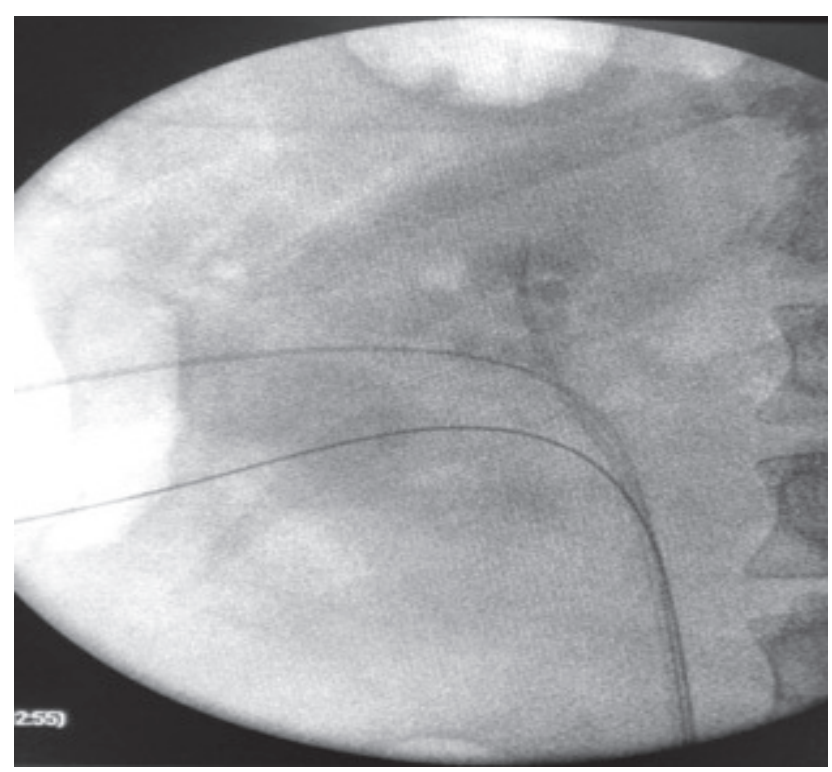

Fig.-4: $2^{\text {nd }}$ guidewire introduced 


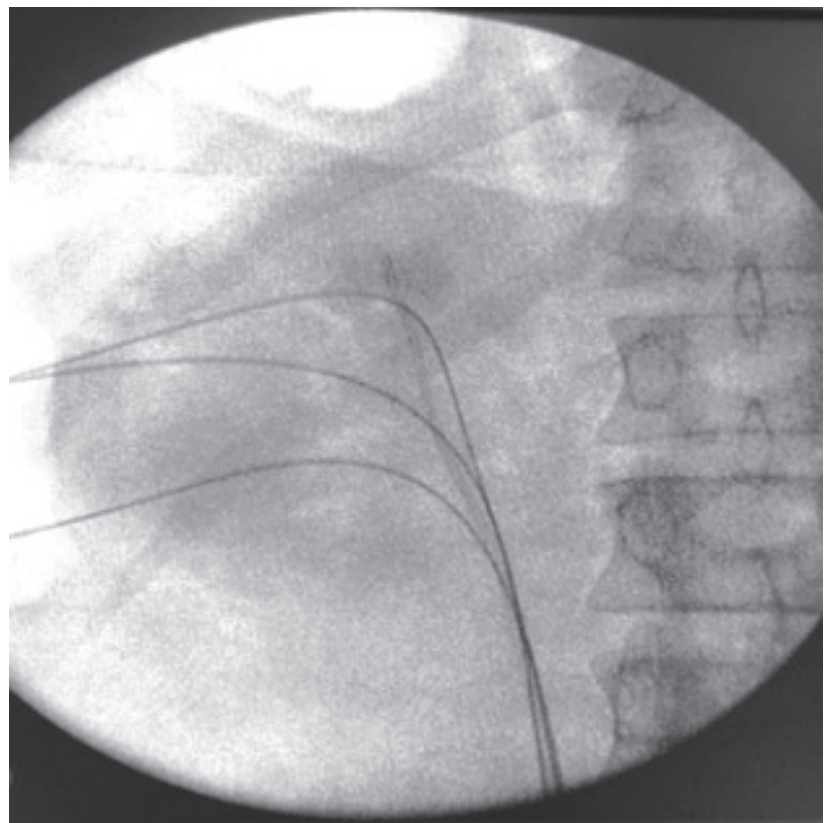

Fig.-5: Third guidewire introduced

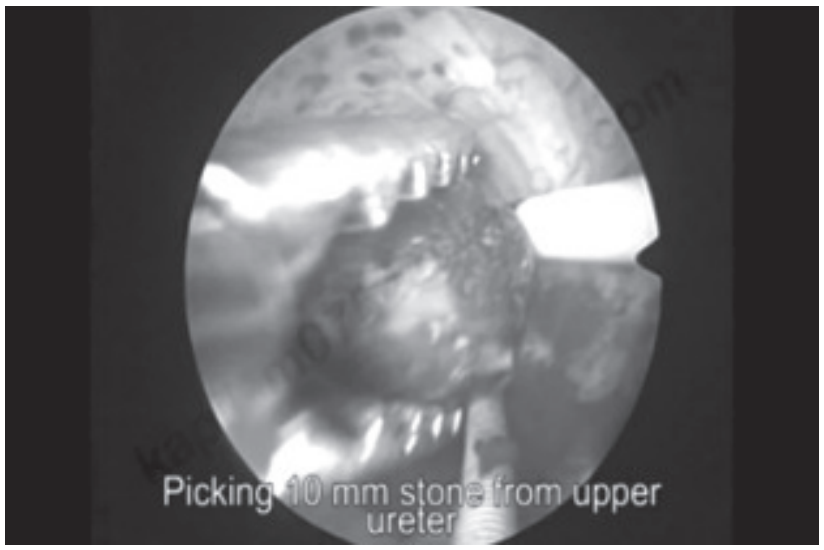

Fig.-7: Picking of stone with forceps

Once the collecting system was entered, a guidewire was passed into the ureter. For patients in whom we used to pass a second guidewire at this stage of the procedure to be used later for the creation of a second access tract.

Subsequently the access tract was dilated with fascial dilators, and then continuing with the standard Amplatz until an Amplatz sheath of 28- $30 \mathrm{Fr}$ was placed. The stones were extracted by an alligator grasper or disintigrated by a standard pneumatic lithoclast before extraction by a grasper. After stone removal, careful inspection of the accessible calices, the renal pelvis, and the UPJ was performed to exclude the presence of residual stones, injury, edema of the UPJ.

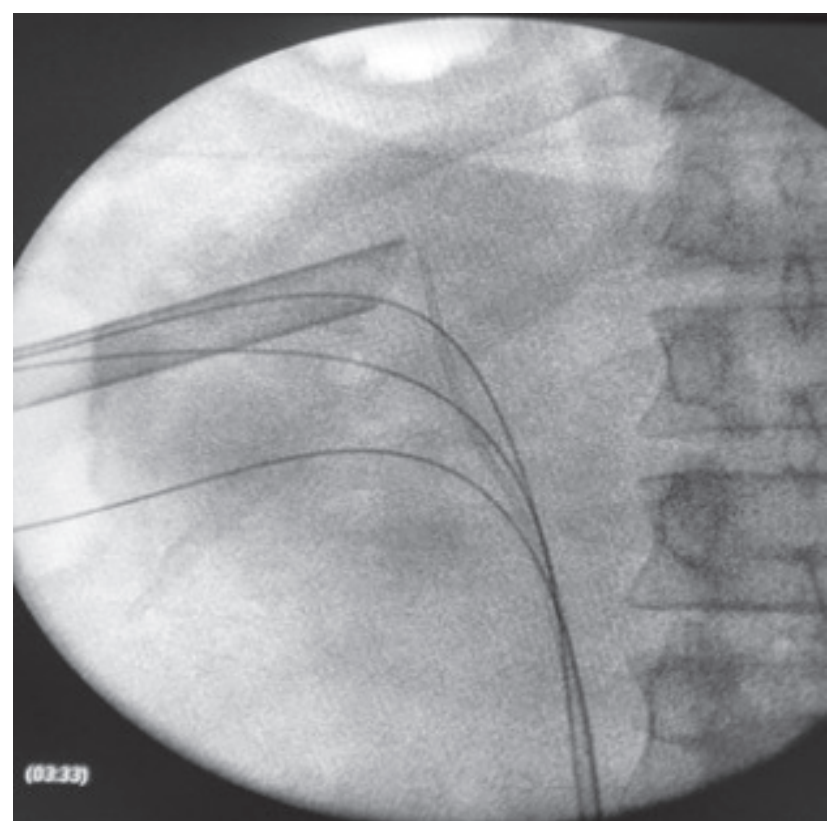

Fig.-6: PCNL in progress

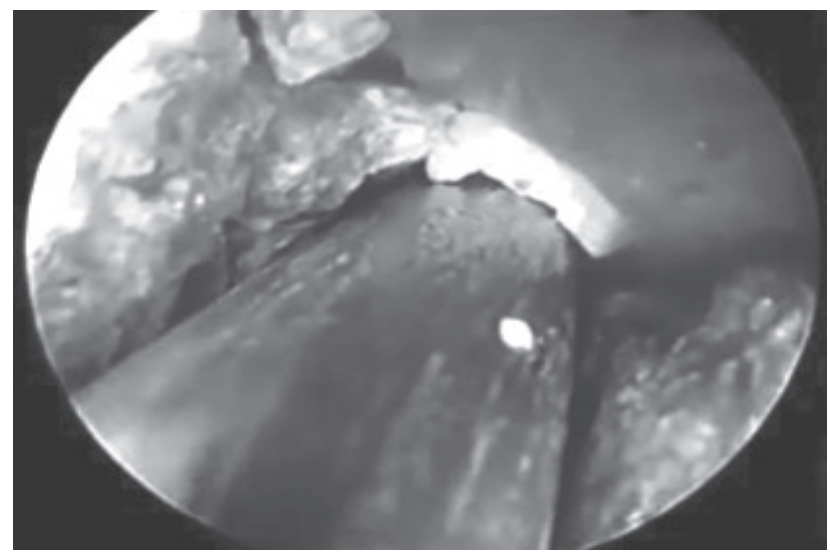

Fig.-8: Stone breaking with lithoclast

A 24 or $28 \mathrm{fr}$ chest drain tube was used to temponade the bleeding, and a 5 or $6 \mathrm{Fr}$ DJ stent was placed in antegradefashioin. On suspicion of chest complication,a thoracic surgeon was consulted and intercostal chest tube drainage was done if indicated.

All patints were examined at the end of the procedure for equal air entry into both sides of the chest, and adequateoxygen saturation was confirmed.In suspicion, Chest radiography was immediately performed after surgery to exclude pneumothorax or hydrothorax. In the immediate post operative period, the patients were observed for signs of bleeding by recording the vital signs and observing the urine output for gross hematuria. Pre and post operativeHb level 
Safety \& Efficacy of Supracostal Access for PCNL: NIKDU Experience

was measured, and any drop was recorded.Stone clearance was assessed by X- Ray KUB and USG of KUB 30 days after surgery. Successful stone clearance was defined as complete clearance of stone or residual fragments $<5 \mathrm{~mm}$.

\section{Results :}

Out of 56 patients $39(70 \%)$ were male. Single tract access was used in $44(78 \%)$ cases, $12(22 \%)$ patients required a second tract. Additional punctures were needed for staghorn calculi (4 out of 14). Overall 82\% of the patients were rendered stone free or had a clinically insignificant residual calculi with PCNL alone and this increases to $96 \%$ with ancillary procedures. In patient with staghorn calculi, they were completely cleared in $78 \%$. Overall complication rate was $26 \%$ which included hydrothorax in $5(10 \%)$ patients, 3 of which required insertion of chest tube. 2 (3.5\%) patient developed haemothorax due toinjury to intercostal vessels, $2(3.5 \%)$ patient developed perforation of the pelvis, $2(3.5 \%)$ developed perinephric collection, infection/ urosepsis in $4(7 \%)$. The rest of the patients recovered successfully. Post operative stay ranged from 2 to 9 days.

Table-I

Indication of Supracostal Puncture

\begin{tabular}{lcc}
\hline Indication & Frequency & Percentage \\
\hline Large stone burden & 22 & $39.28 \%$ \\
Upper calyceal stone & 20 & $35.72 \%$ \\
Upper ureteric stone & 10 & $17.85 \%$ \\
Narrow neck of lower calyx & 2 & $3.57 \%$ \\
High lying kidney & 2 & $3.57 \%$ \\
\hline
\end{tabular}

Table-II

Site of puncture

\begin{tabular}{lc}
\hline Site of puncture & Number (\%) \\
\hline Supracostal access & 56 \\
Calyceal puncture & \\
Superior calyx & $38(68)$ \\
Middle calyx & $16(28.5)$ \\
Lower calyx & $2(3.5)$ \\
\hline
\end{tabular}

Table-III

Complications of the supracostalaccess

\begin{tabular}{lc}
\hline Complications & Number ( \% ) \\
\hline Hydrothoraxx & $5(8.9)$ \\
Hemothorax & $2(3.5)$ \\
Pelvic perforation & $2(3.5)$ \\
Perinephric collection & $2(3.5)$ \\
Infection / Sepsis & $4(7)$ \\
\hline Total & $15(26)$ \\
\hline
\end{tabular}

\section{Discussion :}

We have evaluated 56 cases of supracostal PCNL retrospectively for this study to know the safety profile of supracostal PCNL in terms of hospital stay, intrathoraciccomplications, organ injury, need for blood transfusion, stone clearance rate.

The outcome of PCNL is directly related to an optimal access tract. The superior calyx is considered ideal for approaching the renal system when managing staghorn calculi because the most posterior portion of the kidney is the posterior upper pole calyx, and thus it provides the most direct access to the renal pelvis, upper ureter, and lower pole calyces. ${ }^{6}$ Even the posterior interpolar calyx can be accessible without significant angulation. The superior calyx lie above the $12^{\text {th }}$ rib in most of the times. On full expiration $80 \%$ of the right renal upper pole calyx lie above the $12^{\text {th }}$ rib. Thus direct access to the superior calyx would require a supracostal approach in $>80 \%$ cases. $^{7}$

The subcostal inferior calyx approach to staghorn calculus can induce angulation and torque on the kidney, which can cause trauma and bleeding. ${ }^{8}$ Although it is technically demanding, access through superior calyx provides short and straight access along the axis of the kidney. ${ }^{9}$

In this study,major indications of supracostal puncture were large stone burden,uppercalyceal stone and upper ureteric stone which was consistent with other studies. ${ }^{10,11}$ In the present study, the commonest site of puncture was superior calyx,followed by middle and inferior calyx, which is consistent with other studies. ${ }^{12,13}$

A staghorn calculus was identified as an independent risk factor for severe bleeding after PCNL. 9,10 Preoperative planning of the number, site and direction of the access tract should be determined after a 
thorough evaluation of all radiological studies. ${ }^{14}$ The surgeon must get a balance between complete stone clearance and acceptable patient morbidity. Therefore when significant morbidity develops, the procedure should be terminated. ${ }^{15}$

In the present study significant blood loss, which require blood transfusions in $14.7 \%$ of the cases. These results were similar with the other studies. ${ }^{11} \mathrm{This}$ high rate of excessive bleeding was because the vascularity of the upper pole and injury to the intercostals vessels.

Overall complication rate in the present study was similar to other studies. ${ }^{15,16}$

\section{Conclusion:}

Establishing good percutaneous renal access is a crucial step in PCNL technique.Supracostal approach can be ideal for many clinical situations. When the supracostal approach is used complications may be limited by positioning the Amplatz sheath well into the collecting system, puncture should be made during breath holding in full expiration, and should avoid the lower rib margin to avoid the intercostals vessels and should always be in the lateral half of the ICS. With a good understanding of the anatomy surrounding the upper pole of the kidney and attention to a few technical considerations during the procedure upper calyceal approach can be performed safely and effectively.

\section{References:}

1. Golijanin, D.,Katz, R., Verstandig, A. et al. The supracostal nephrostomy for treatment of staghorn and complex kidney stones. J Endourol 1998;12:403-405

2. Miller, N.L., Matlage, B.R., Lingemen, J.E. Techniques for fluoroscopic percutaneous renal access. J Urol2007; 178:15-23.

3. Sukumar, S.,Nair, B., Kumar, P.G. et al. Supracostal access nephrostomy : Less morbid more effective . IntUroINephrol2008;40:263-267.

4. Yadav, R., Gupta, N.P., Gamanaagatti, S. et al. Supra-twelfth supacostal access: When and where to puncture? J Endourol2008; 22: 1-4

5. Maheshwari, P.N., Mane, D.A., Pathak, A.B. Management of pleural injury after percutaneous renal surgery. J Endourol2009; 23: 1769-1772.

6. E.Lang, R.Thomas, R. Davis, I.Colon, M.Allaf, A. Hanano, et al. Risks, advantages and complications of intercostal vs subcostal approach for percutaneous nephrostomy Urology. 47(2009), pp.751-756.

7. P.B. Irby, B.F. Schwartz. M.L. Stoller Percutaneous access techniques in renal surgery Tech Urol. 5(1999).pp29-39.

8. Morris, D.S., Wei, J.T., Taub, D.A., Dunn, R.L., Wolf, J.S. Jr, Hollenbeck, B.K. Contemporary trends in the use of percutaneous nephrostomy. J Urol2006; 175:1731-1736.

9. Kekre, N.S., Gopalakrishnan, G.G., Gupta, G.G. et al. Supracostal approach in percutaneous nephrostomy: experience with 102 cases. J EndoUrol2001;15:789-791.

10. A.R. El-Nahas, A.A Shokeir, A.M. El- Assmy, T. Mohsen , A.M Shoma , L.Eraky, et al. Postpercutaneous nephrostomy extensive hemorrhage. A study of risk factors $\mathrm{J}$ Urol177(2007). pp 576-579.

11. A.R,El-Nahas, I.Eraky ,A.A. Shokeir. A.M Shoma, A.M. El- Assmy, N.A. El- Tabey, et al. Factors affecting stone-free rate and complications of percutaneous nephrostomy for treatment of staghorn stone Urology. 79(2012). pp. 1236-1241.

12. A.R. El-Nahas,I. Eraky, A. A. Shokeir. A.M Shoma, A.M. El- Assmy, N.A. El- Tabey, et al. Percutaneous nephrostomy for treating staghorn stones. 10 years of experience of a tertiary-care center. Arab .J Urol10(2012). Pp, 324-329.

13. Gupta R., Kumar. A., Kapoor, R. et al. Prospective evaluation of safety and efficacy of the supracostal approach for percutaneous nephrostomy. BJU Int2002; 90:809-813.

14. Lojanapiwat, B., Prasopsuk, S. Upper-pole access for percutaneous nephrostomy: comparison of supracostal and infracostalapproaches. J Endourol 2006;20:491-494.

15. Shah, H.N., Hegde, S.S. Hah, J.H. et al. Safety and efficacy of supracostal access in tubeless percutaneous nephrostomy. iEndourol 2006; 20: 1016-1021.

16. Shaban, A., Kodera, A., Elghoneimy, M.N, et al. Safety and efficacy of supracostal access in percutaneous renal surgery. J Endourol2008; 22:29-33. 